\title{
Standard molar enthalpy of formation of 2,4,6-trimethylpyridine
}

\author{
Manuel A. V. Ribeiro da Silva, ${ }^{a}$ M. Agostinha R. Matos, \\ and Carolina M. A. do Rio \\ Centro de Investigação em Química, Department of Chemistry, \\ Faculty of Science, University of Porto, Rua do Campo Alegre, 687, \\ P-4150 Porto, Portugal
}

\begin{abstract}
The standard $\left(p^{\circ}=0.1 \mathrm{MPa}\right.$ ) molar enthalpy of formation for liquid 2,4,6-trimethylpyridine was derived from the standard molar enthalpy of combustion, measured by static bomb combustion calorimetry in oxygen, at the temperature $T=298.15 \mathrm{~K}: \Delta_{\mathrm{f}} H_{\mathrm{m}}^{\circ}=$ $-(31.0 \pm 2.3) \mathrm{kJ} \cdot \mathrm{mol}^{-1}$. (c) 1997 Academic Press Limited
\end{abstract}

KEYWORDS: enthalpy of formation; 2,4,6-trimethylpyridime; 2,4,6-collidine; combustion calorimetry

\section{Introduction}

Recently, we reported thermochemical and theoretical studies on methyldiazines ${ }^{(1)}$ as part of our broader study on the thermochemical properties of nitrogen heterocycles. $^{(2-11)}$ The results have been discussed in terms of the enthalpic contribution of the substituents, and it was found ${ }^{(1)}$ that, although the enthalpic increment for the tetramethylation of pyrazine was twice that for the dimethylation, this simple scheme of additivity did not work for the trimethylation. In view of the literature results available for the standard molar enthalpies of formation $\Delta_{\mathrm{f}} H_{\mathrm{m}}^{\circ}$ for monomethyl and dimethylpyridines, it was decided to study the thermochemistry of 2,4,6-trimethylpyridine (2,4,6-collidine), in order to analyse how the additivity schemes worked for di- and trimethylation of pyridines.

This paper reports the standard $\left(p^{\circ}=0.1 \mathrm{MPa}\right)$ molar enthalpy of combustion for that compound in oxygen at the temperature $T=298.15 \mathrm{~K}$, measured by static-bomb combustion calorimetry, and the derived value for the standard molar enthalpy of formation in the liquid phase. Using this last value and the literature ${ }^{(13)}$ value for the standard molar enthalpy of vaporization at the temperature of $298.15 \mathrm{~K}$, we have calculated the value for the standard molar enthalpy of formation for 2,4,6-trimethylpyridine in the gaseous state. This value is compared with those for other methyl-substituted pyridines and pyrazines.

\footnotetext{
${ }^{a}$ To whom correspondence should be addressed.
} 


\section{Experimental}

The 2,4,6-trimethylpyridine, obtained from Aldrich Chemical Co., was purified by repeated vacuum distillation until the combustion results were consistent and the carbon dioxide recovery ratios were satisfactory. Purity was confirmed by elemental microanalysis and i.r. spectroscopy. The average ratio, together with the standard deviation of the mean, of the mass of carbon dioxide recovered to that calculated from the mass of sample was $(1.0000 \pm 0.0007)$.

The combustion experiments were performed with a static bomb calorimeter; the apparatus and technique have been described previously. ${ }^{(14,15)}$ The energy equivalent of the calorimeter was determined from the combustion of benzoic acid (Bureau of Analysed Samples, Thermochemical Standard, BAS-CRM-190 p), having a massic energy of combustion, under standard bomb conditions, of $-(26431.8 \pm 3.7) \mathrm{J} \cdot \mathrm{g}^{-1}$. The calibration results were corrected to give the energy equivalent $\varepsilon$ (calor) corresponding to the average mass of water added to the calorimeter: $3119.6 \mathrm{~g}$. From nine calibration experiments, $\varepsilon$ (calor) $=$ $(15911.2 \pm 1.5) \mathrm{J} \cdot \mathrm{K}^{-1}$, where the uncertainty quoted is the standard deviation of the mean.

The liquid samples were contained in sealed polyester bags made from Melinex $(0.025 \mathrm{~mm}$ thickness) using the technique described by Skinner and Snelson ${ }^{(16)}$ who determined the standard massic energy of combustion of dry Melinex as $-\Delta_{\mathrm{c}} u^{\circ}=(22902 \pm 5) \mathrm{J} \cdot \mathrm{g}^{-1}$; this value has been confirmed by combustion of Melinex samples in our laboratory. The mass of Melinex used in each experiment was corrected for the mass fraction of water $(x=0.0032)$, and the mass of carbon dioxide produced from the Melinex was calculated using the factor previously reported. ${ }^{(16)}$ Combustion experiments were made in oxygen at $p=3.04 \mathrm{MPa}$, with $1 \mathrm{~cm}^{3}$ of water added to the bomb. For all experiments, ignition was made at $T=(298.150 \pm 0.001) \mathrm{K}$. The electrical energy for ignition was determined from the change in potential difference across a capacitor when discharged through the platinum ignition wire. For the cotton thread fuse, empirical formula $\mathrm{CH}_{1.686} \mathrm{O}_{0.843}$, the standard massic energy of combustion is $\Delta_{\mathrm{c}} u^{\circ}=-16250 \mathrm{~J} \cdot \mathrm{g}^{-1} \cdot{ }^{(17)}$ This value has been confirmed by previous combustion of cotton-thread samples in our laboratory. Corrections for nitric acid formation were based on $-59.7 \mathrm{~kJ} \cdot \mathrm{mol}^{-1}$ for the molar energy of formation of $0.1 \mathrm{~mol} \cdot \mathrm{dm}^{-3} \mathrm{HNO}_{3}(\mathrm{aq})$ from $(1 / 2) \cdot \mathrm{N}_{2}(\mathrm{~g}),(5 / 4) \cdot \mathrm{O}_{2}(\mathrm{~g})$, and $(1 / 2) \mathrm{H}_{2} \mathrm{O}(1) \cdot{ }^{(18)}$ Corrections for carbon formation were based on the standard massic energy of combustion of carbon $\Delta_{\mathrm{c}} u^{\circ}=-33 \mathrm{~kJ} \cdot \mathrm{g}^{-1}$. $^{(19)}$ From the nine combustion experiments performed, only one had a very small amount of carbon residue. The absence of carbon monoxide in the combustion gases was tested by means of Winkler's reagent. The amount of substance used in each experiment was determined from the total mass of carbon dioxide produced after allowance for that formed from the cotton thread fuse and from the Melinex bag, and that lost due to carbon formation. The density of 2,4,6-trimethylpyridine $\left(2,4,6\right.$-collidine) is $0.917 \mathrm{~g} \cdot \mathrm{cm}^{-3} \cdot{ }^{(20)}$ An estimated pressure coefficient of massic energy: $(\partial u / \partial p)_{T}=-0.2 \mathrm{~J} \cdot \mathrm{g}^{-1} \cdot \mathrm{MPa}^{-1}$ at $T=298.15 \mathrm{~K}$, a typical value for most organic compounds, was assumed. The standard massic energy of combustion $\Delta_{\mathrm{c}} u^{\circ}$ for 2,4,6-trimethylpyridine was 
TABLE 1. Typical combustion experiment of 2,4,6-trimethylpyridine at $T=298.15 \mathrm{~K}$

\begin{tabular}{lc}
\hline$m\left(\mathrm{CO}_{2}\right.$, total $) / \mathrm{g}$ & 2.02720 \\
$m^{\prime}(\mathrm{cpd}) / \mathrm{g}$ & 0.66401 \\
$m^{\prime \prime}($ fuse $) / \mathrm{g}$ & 0.00417 \\
$m^{\prime \prime \prime}($ Melinex $) / \mathrm{g}$ & 0.03984 \\
$\Delta T_{\mathrm{ad}} / \mathrm{K}$ & 1.67565 \\
$\varepsilon_{\mathrm{f}} /\left(\mathrm{J} \cdot \mathrm{K}^{-1}\right)$ & 16.71 \\
$\Delta m\left(\mathrm{H}_{2} \mathrm{O}\right) / \mathrm{g}$ & 0.1 \\
$-\Delta U(\mathrm{IBP}) / \mathrm{J}$ & 26689.11 \\
$\left.\Delta U(\mathrm{HNO})_{3}\right) / \mathrm{J}$ & 37.55 \\
$\Delta U(\mathrm{ign}) / \mathrm{J}$ & 1.19 \\
$\Delta U_{\Sigma} / \mathrm{J}$ & 13.41 \\
$\Delta U($ carbon $) / \mathrm{J}$ & 0.00 \\
$-\Delta U(\mathrm{Melinex}) / \mathrm{J}$ & 912.42 \\
$-\Delta U($ fuse $) / \mathrm{J}$ & 67.72 \\
$-\Delta_{\mathrm{c}} u^{\circ} /\left(\mathrm{J} \cdot \mathrm{g}^{-1}\right)$ & 38641.00 \\
\hline
\end{tabular}

calculated using the procedure given by Hubbard et al. ${ }^{(17)}$ The relative atomic masses used were those recommended by the IUPAC Commission. ${ }^{(21)}$

\section{Results}

Results for a typical combustion experiment are given in table 1, where $\Delta m\left(\mathrm{H}_{2} \mathrm{O}\right)$ is the deviation of the mass of water added to the calorimeter from $3119.6 \mathrm{~g}$. Samples were ignited at $T=298.15 \mathrm{~K}$ so that $\Delta U(\mathrm{IBP})=$ $-\left\{\varepsilon\right.$ (calor) $\left.+\Delta m\left(\mathrm{H}_{2} \mathrm{O}\right) \cdot c_{p}\left(\mathrm{H}_{2} \mathrm{O}, 1\right)+\varepsilon_{f}\right\} \cdot \Delta T_{\text {ad }}+\Delta U_{\text {ign. }}$. In table $1, \Delta U_{\Sigma}$ is the correction to the standard state; the remaining quantities are as previously defined. ${ }^{(17)}$

The individual results of all nine combustion experiments, together with the mean value and its standard deviation, are given in table 2 . Table 3 lists the derived standard molar energy and enthalpy of combustion and the standard molar enthalpy of formation for 2,4,6-trimethylpyridine in the liquid state at $\mathrm{T}=298.15 \mathrm{~K}$. In accordance with normal thermochemical practice, the uncertainty assigned to the standard molar enthalpy of combustion is twice the overall standard deviation of the mean, and includes the uncertainties in calibration and in the values

TABLE 2. Individual values of the standard massic energy of combustion $\Delta_{\mathrm{c}} u^{\circ}$ of 2,4,6-trimethylpyridine, at $T=298.15 \mathrm{~K}$

\begin{tabular}{rr}
\hline \multicolumn{3}{c}{$-\Delta_{\mathrm{c}} \mathcal{u}^{\circ} /\left(\mathrm{J} \cdot \mathrm{g}^{-1}\right)$} \\
\hline 38676.32 & 38652.17 \\
38639.21 & 38627.06 \\
38641.00 & 38666.19 \\
38657.90 & 38637.77 \\
38638.57 & \\
$-\left\langle\Delta_{\mathrm{c}} \mathcal{u}^{\circ}\right\rangle=(38648.5 \pm 4.3) \mathrm{J} \cdot \mathrm{g}^{-1}{ }^{a}$ \\
\hline
\end{tabular}

\footnotetext{
${ }^{a}$ The uncertainty is the standard deviation of the mean.
} 
TABLE 3. Derived standard $\left(p^{\circ}=0.1 \mathrm{MPa}\right)$ molar values for 2,4,6-trimethylpyridine in the liquid state at $T=298.15 \mathrm{~K}$, where $\Delta_{\mathrm{c}} U_{\mathrm{m}}^{\circ}$ is the standard molar energy of combustion, $\Delta_{\mathrm{c}} H_{\mathrm{m}}^{\circ}$ is the standard molar enthalpy of combustion, and $\Delta_{\mathrm{f}} H_{\mathrm{m}}^{\circ}$ is the standard molar enthalpy of formation

\begin{tabular}{ccc}
\hline$\Delta_{\mathrm{c}} U_{\mathrm{m}}^{\circ} /\left(\mathrm{kJ} \cdot \mathrm{mol}^{-1}\right)$ & $\Delta_{\mathrm{c}} H_{\mathrm{m}}^{\circ} /\left(\mathrm{kJ} \cdot \mathrm{mol}^{-1}\right)$ & $\Delta_{\mathrm{f}} H_{\mathrm{m}}^{\circ}(\mathrm{l}) /\left(\mathrm{kJ} \cdot \mathrm{mol}^{-1}\right)$ \\
\hline$-4683.5 \pm 2.0$ & $-4689.1 \pm 2.0$ & $-31.0 \pm 2.3$ \\
\hline
\end{tabular}

of the auxiliary quantities used. ${ }^{(22)}$ To derive $\Delta_{\mathrm{f}} H_{\mathrm{m}}^{\circ}(1)$ from $\Delta_{\mathrm{c}} H_{\mathrm{m}}^{\circ}$, the standard molar enthalpies of formation of $\mathrm{H}_{2} \mathrm{O}(\mathrm{l})$ and $\mathrm{CO}_{2}(\mathrm{~g})$ at $T=298.15 \mathrm{~K}$, $-(285.830 \pm 0.042) \mathrm{kJ} \cdot \mathrm{mol}^{-1(23)}$ and $-(393.51 \pm 0.13) \mathrm{kJ} \cdot \mathrm{mol}^{-1},{ }^{(23)}$ respectively, were used.

\section{Discussion}

The standard molar enthalpy of vaporization of 2,4,6-trimethylpyridine at $T=298.15 \mathrm{~K}, \Delta_{\mathrm{l}}^{\mathrm{g}} H_{\mathrm{m}}^{\circ}(298.15 \mathrm{~K})=(50.33 \pm 0.15) \mathrm{kJ} \cdot \mathrm{mol}^{-1}$, was determined by Majer et al. ${ }^{(13)}$ in 1985. Using this value, together with the standard molar enthalpy of formation in the liquid state, the standard molar enthalpy of formation in the gaseous state is derived as $(19.30 \pm 2.4) \mathrm{kJ} \cdot \mathrm{mol}^{-1}$.

From the available values for the standard molar enthalpies of formation of pyrazine and methylsubstituted pyrazines, which are shown in table 4 , we concluded in a previous paper ${ }^{(1)}$ that the $\Delta_{\mathrm{r}} H_{\mathrm{m}}^{\circ}$ for the tetramethylation of pyrazine, $-(141.3 \pm 4.7) \mathrm{kJ} \cdot \mathrm{mol}^{-1}$, is twice that verified for the dimethylation, $-(70.0 \pm 5.2) \mathrm{kJ} \cdot \mathrm{mol}^{-1}$, as shown in table 4. However, as we previously

TABLE 4. Standard molar enthalpies of formation of some pyridines and pyrazines in the gaseous state at $T=298.15 \mathrm{~K}$, and the enthalpy increments $\Delta_{\mathrm{r}} H_{\mathrm{m}}^{\circ}$ due to successive methyl substitutions

\begin{tabular}{lrrr} 
& $\Delta_{\mathrm{f}} H_{\mathrm{m}}^{\circ} /\left(\mathrm{kJ} \cdot \mathrm{mol}^{-1}\right)$ & \multicolumn{2}{c}{$\Delta_{\mathrm{r}} H_{\mathrm{m}}^{\circ} /\left(\mathrm{kJ} \cdot \mathrm{mol}^{-1}\right)^{a}$} \\
\cline { 3 - 4 } Compound & & experimental & estimated \\
\hline Pyridine & $140.4 \pm 0.7^{(12)}$ & & \\
2-Methylpyridine & $99.2 \pm 0.7^{(12)}$ & $-41.2 \pm 1.0$ & \\
3-Methylpyridine & $106.5 \pm 0.6^{(12)}$ & $-33.9 \pm 0.9$ & \\
4-Methylpyridine & $104.0 \pm 0.9^{(12)}$ & $-73.3 \pm 1.1$ & $-75.1 \pm 1.3$ \\
2,3-Dimethylpyridine & $67.1 \pm 1.3^{(12)}$ & $-76.8 \pm 1.1$ & $-77.6 \pm 1.5$ \\
2,4-Dimethylpyridine & $63.6 \pm 0.8^{(12)}$ & $-73.9 \pm 1.2$ & $-75.1 \pm 1.3$ \\
2,5-Dimethylpyridine & $66.5 \pm 1.0^{(12)}$ & $-82.3 \pm 1.7$ & $-82.4 \pm 1.4$ \\
2,6-Dimethylpyridine & $58.1 \pm 1.5^{(12)}$ & $-68.4 \pm 1.1$ & $-70.3 \pm 1.4$ \\
3,4-Dimethylpyridine & $68.8 \pm 1.0^{(12)}$ & $-121.1 \pm 2.5$ & $-67.8 \pm 1.3$ \\
3,5-Dimethylpyridine & $72.0 \pm 0.9^{(12)}$ & & \\
2,4,6-Trimethylpyridine & $19.3 \pm 2.4$ & $-70.0 \pm 5.2$ & \\
Pyrazine & $196.0 \pm 1.5^{(24)}$ & $-121.7 \pm 3.1$ & $-105.9 \pm 7.8$ \\
2,3-Dimethylpyrazine & $126.0 \pm 5.0^{(1)}$ & $-141.3 \pm 4.7$ & $-140.0 \pm 7.4$ \\
Trimethylpyrazine & $74.3 \pm 2.7^{(1)}$ & $54.7 \pm 4.5^{(1)}$ & \\
Tetramethylpyrazine & & & \\
\hline
\end{tabular}

${ }^{a} \Delta_{\mathrm{r}} H_{\mathrm{m}}^{\circ}=\Delta_{\mathrm{f}} H_{\mathrm{m}}^{\circ}\left\{(\text { methyl })_{n}\right.$ pyridine, $\left.\mathrm{g}\right\}-\Delta_{\mathrm{f}} H_{\mathrm{m}}^{\circ}($ pyridine, g $)$, or $\Delta_{\mathrm{r}} H_{\mathrm{m}}^{\circ}=\Delta_{\mathrm{f}} H_{\mathrm{m}}^{\circ}\left\{(\text { methyl })_{n}\right.$ pyrazine, $\left.\mathrm{g}\right\}-$ $\Delta_{\mathrm{f}} H_{\mathrm{m}}^{\circ}\{$ pyrazine, $\mathrm{g}\}, n=$ number of methyl substituents on the molecule. 
reported ${ }^{(1)}$ the standard molar enthalpy of formation of trimethylpyrazine shows that $\Delta_{\mathrm{r}} H_{\mathrm{m}}^{\circ}$, the enthalpy increment for trimethylation of pyrazine, $-(121.7 \pm 3.1) \mathrm{kJ} \cdot \mathrm{mol}^{-1}$, is not simply equal to 0.75 of the tetramethylation enthalpy increment, $-(105.9 \pm 7.8) \mathrm{kJ} \cdot \mathrm{mol}^{-1}$, meaning that the successive methylations of pyrazine are not the sum of individual increments alone. This experimental fact is confirmed by the theoretical estimates of the electronic energy, using the Density Functional Theory (DFT) ${ }^{(1)}$ which correlate well with the experimental values for the standard molar enthalpies of formation of the three methylated pyrazines studied.

The $\Delta_{\mathrm{r}} H_{\mathrm{m}}^{\circ}$ of methylations of pyridine can be examined from the available values for the standard molar enthalpies of formation of monomethylpyridines and dimethylpyridines which are shown in table 4 . The $\Delta_{\mathrm{r}} H_{\mathrm{m}}^{\circ}$ for the monomethylation of pyridine in the ortho position is $-(41.2 \pm 1.0) \mathrm{kJ} \cdot \mathrm{mol}^{-1}$; in the meta position, it is $-(33.9 \pm 0.9) \mathrm{kJ} \cdot \mathrm{mol}^{-1}$; and in the para position, it is $-(36.4 \pm 1.1) \mathrm{kJ} \cdot \mathrm{mol}^{-1}$. Using these given $\Delta_{\mathrm{r}} H_{\mathrm{m}}^{\circ} \mathrm{S}$ values to calculate the $\Delta_{\mathrm{r}} H_{\mathrm{m}}^{\circ}$ for dimethylpyridines, one can conclude that these estimated values reproduce, within the experimental associated uncertainties, the derived values from the experimentally obtained $\Delta_{\mathrm{f}} H_{\mathrm{m}}^{\circ}(\mathrm{g})$ in all cases, even when both methyl groups enter in the ortho position, one relative to the other (see table 4).

The experimental value obtained in this work for the standard molar enthalpy of formation of 2,4,6-trimethylpyridine is equal, within the experimental uncertainty, to the result we could derive from the enthalpy of formation of pyridine, adding the $\Delta_{\mathrm{r}} H_{\mathrm{m}}^{\circ}$ twice due to the ortho methylation to the value for para substitution (see table 4). It can therefore be concluded that in pyridine there is transferability of group enthalpy contributions for methyl substitutions, which is not the case for pyrazine. This may be due to the presence of a second nitrogen atom in the pyrazine ring.

Thanks are due to Junta Nacional de Investigação Científica e Tecnológica (JNICT) for financial support of the Project PBIC/C/CEN/1019/92, as well as to Centro de Investigação em Química, University of Porto (Q.P./1-L.5). C. M. A. Rio thanks JNICT for the award of a research grant under PRAXIS XXI (research grant $\mathrm{BM} / 2003 / 94)$.

\section{REFERENCES}

1. Ribeiro da Silva, M. A. V.; Morais, V. M. F.; Matos, M. A. R.; Rio, C. M. A.; Piedade, C. M. G. S. Struct. Chem. 1996, 7, 329-336.

2. Ribeiro da Silva, M. A. V.; Monte, M. J. S.; Matos, M. A. R. J. Chem. Thermodynamics 1989, 21, 159-166.

3. Ribeiro da Silva, M. A. V.; Matos, M. A. R.; Monte, M. J. S. J. Chem. Thermodynamics 1990, 22, 609-616.

4. Ribeiro da Silva, M. A. V.; Monte, M. J. S. J. Chem. Thermodynamics 1992, 24, 715-724.

5. Ribeiro da Silva, M. A. V.; Matos, M. A. R.; Monte, M. J. S.; Alves, M. C. B.; Vieira, J. M. A. P. J. Chem. Thermodynamics 1993, 25, 597-590.

6. Ribeiro da Silva, M. A. V.; Ferrão, M. L. C. H.; Silva, A. M. R. O. A. J. Chem. Thermodynamics 1995, 27, 633-641. 
7. Ribeiro da Silva, M. A. V.; Matos, M. A. R.; Amaral, L. M. P. F. J. Chem. Thermodynamics 1995, 27, 565-574.

8. Ribeiro da Silva, M. A. V.; Matos, M. A. r.; Amaral, L. M. P. F. J. Chem. Thermodynamics 1995, 27, 1141-1145.

9. Ribeiro da Silva, M. A. V.; Matos, M. A. R.; Amaral, L. M. P. F. J. Chem. Thermodynamics 1995, 27, 1187-1196.

10. Ribeiro da Silva, M. A. V.; Morais, V. M. F.; Matos, M. A. R.; Rio, C. M. A. J. Org. Chem. 1995, 60, 5291-5294.

11. Ribeiro da Silva, M. A. V.; Morais, V. M. F.; Matos, M. A. R. J. Chem. Soc. Faraday Trans. 1995, 92, 1907-1910.

12. Pedley, J. B. Thermochemical Data and Structures of Organic Compounds, Vol.1 TRC Data Series: College Station, TX. 1994.

13. Majer, V.; Svoboda, V.; Lencka, M. J. Chem. Thermodynamics 1985, 17, 365-370.

14. Ribeiro da Silva, M. A. V.; Ribeiro da Silva, M. D. M. C.; Pilcher, G. Rev. Port. Quim. 1984, 26, $163-172$.

15. Ribeiro da Silva, M. A. V.; Ribeiro da Silva, M. D. M. C.; Pilcher, G. J. Chem. Thermodynamics 1984, 16, 1149-1155.

16. Skinner, H. A.; Snelson, A. Trans. Faraday Soc. 1960, 56, 1776.

17. Hubbard, W. N.; Scott, D. W.; Waddington, G. Experimental Thermochemistry, Vol.1. Rossini, F. D.: editor. Interscience: New York. 1956, Chap. 5.

18. The NBS Tables of Chemical Thermodynamic Properties. J. Phys. Chem. Ref. Data 1982, 11, Suppl. 2.

19. Coops, J.; Jessup, R. S.; Van Nes, K. Experimental Thermochemistry, Vol.1. Rossini, F. D.: editor. Interscience: New York. 1956, Chap. 3.

20. Handbook of Chemistry and Physics, 70th edition. Weast, R. C.: editor. CRC Press: Boca Raton, FL. 1989-1990.

21. IUPAC. J. Phys. Chem. Ref. Data 1993, 22, 1571-1584.

22. Rossini, F. D. Experimental Thermochemistry, Vol.1. Rossini, F. D.: editor. Interscience: New York 1956, Chap. 14.

23. J. Chem. Thermodynamics 1978, 10, 903-906.

24. Tjebbes, J. Acta Chem. Scand. 1962, 16, 916-921.

(Received 9 December 1996; in final form 18 February 1997)

O-659 